\title{
Incidence and Temporal Changes in Lumbar Degeneration and Low Back Pain in Child and Adolescent Weightlifters: Prospective 5-Year Cohort Study
}

\section{Rikuto Yoshimizu}

Department of Orthopaedic Surgery, Graduate School of Medical Science, Kanazawa University Junsuke Nakase ( $\square$ nakase1007@yahoo.co.jp)

Department of Orthopaedic Surgery, Graduate School of Medical Science, Kanazawa University Katsuhito Yoshioka

Department of Orthopedic Surgery, National Hospital Organization Kanazawa Medical Center.

\section{Kengo Shimozaki}

Department of Orthopaedic Surgery, Graduate School of Medical Science, Kanazawa University

\section{Kazuki Asai}

Department of Orthopaedic Surgery, Graduate School of Medical Science, Kanazawa University

\section{Kazu Toyooka}

Department of Orthopaedic Surgery, Graduate School of Medical Science, Kanazawa University

\section{Mitsuhiro Kimura}

Department of Orthopaedic Surgery, Graduate School of Medical Science, Kanazawa University

\section{Katsuhiko Kitaoka}

Department of Orthopedic Surgery, Kijima Hospital.

\section{Hiroyuki Tsuchiya}

Department of Orthopaedic Surgery, Graduate School of Medical Science, Kanazawa University

\section{Research Article}

Keywords: Lumbar Degeneration, Adolescent Weightlifters, Cohort Study

Posted Date: May 20th, 2021

DOl: https://doi.org/10.21203/rs.3.rs-533539/v1

License: (c) (1) This work is licensed under a Creative Commons Attribution 4.0 International License. Read Full License 


\section{Abstract}

\section{Purpose}

To investigate the incidence of lumbar degeneration findings and low back pain (LBP) over a 5-year period in children and adolescent weightlifters using magnetic resonance imaging (MRI) and medical questionnaires. Furthermore, to reveal the temporal changes in the lumbar vertebrae caused by long-term hard weightlifting training during the growth period.

\section{Methods}

Twelve children and adolescent weightlifters who participated in weightlifting for $>2$ years (six boys, six girls, $11.4 \pm 2.0$ years) were enrolled. Participants underwent annual medical questionnaire surveys including practice frequency, competition history, presence of LBP, and lumbar examinations using MRI during the 5-year follow-up.

Results

Lumbar disc degeneration was detected in all participants after 4 years, and lumbar disc herniation findings were detected in $33 \%$ of participants after 5 years; one underwent herniotomy during the followup period. Lumbar spondylolysis was detected in $58 \%$ of patients at 5 years. Although there were three participants who had LBP in the final year, none had LBP that prevented them from returning to weightlifting.

\section{Conclusion}

A 5-year cohort study of 12 children and adolescent weightlifters detected lumbar degeneration in all participants. Long-term hard weightlifting training during the growth period may increase the risk of developing current and future LBP.

\section{Introduction}

Weightlifting training is practiced by athletes of all ages to improve performance and prevent injuries [1]. On the other hand, athletes may suffer injuries such as back pain if they do not choose the correct weight and have proper form $[1,2]$. Particularly, weightlifting training in young athletes can damage the growth plate and should be performed with extreme caution [3].

Low back pain (LBP) is one of the most common weightlifting complaints, with incidence rates $\geq 40 \%$, caused by lumbar disc degeneration and herniation, which can interfere with not only sports activities but also social life [2]. Although few studies have investigated whether weightlifting training during the growth period is associated with future lumbar disc degeneration and LBP, it is important to note that spinal abnormalities that develop at a young age not only cause LBP at that time, but also increase the 
risk of recurrence of LBP in adulthood [4,5]. Therefore, it is necessary to pay close attention to weightlifting training during the growth period.

Previous reports have reported a prevalence of $>80 \%$ of radiological changes in the lumbar vertebrae, such as lumbar disc herniation, caused by long-term weightlifting training; however, these reports are from experienced adolescents $[6,7]$. Our 3-year prospective cohort study is the only study to observe radiological changes in the lumbar vertebrae of children and adolescents [8]. In this study of 12 child and adolescent weightlifter participants, 11 participants had detectable lumbar degeneration findings, mainly disc degeneration, and it was concluded that resistance training at the competition level in children and adolescents could cause irreversible changes in the lumbar vertebrae.

The purpose of this study was to investigate the incidence of lumbar degeneration and LBP over a 5-year period in child and adolescent weightlifters using magnetic resonance imaging (MRI) and medical questionnaires. This cohort study revealed the temporal changes in the lumbar vertebrae caused by longterm hard weightlifting training during the growth period that have not been revealed so far.

\section{Methods}

This study was conducted between 2014 and 2018, and 12 participants (six boys and six girls) were enrolled (Table 1). The mean age of participants at the start of the study was $11.4 \pm 2.0$ years and the average competition history was $2.2 \pm 0.7$ years. Most participants also participated in sports other than weightlifting at the start of the study. All participants had no history of lumbar diseases or surgeries and were followed up for 5 years. The study design was approved by the Ethical Committee of the Graduate School of Medical Sciences, Kanazawa University (approval \#1399). The design and purpose of this study were explained to the participants and their parents, and written informed consent was obtained from all participants and their parents. The participants underwent annual medical examinations during the 5-year follow-up period. All human experiments in this study followed the guidelines of the Declaration of Helsinki.

In this study, LBP was defined as a condition in which participants were unable to practice weightlifting for more than 1 week due to pain. All participants adhered to the rule of stopping training when symptoms of LBP appeared and restarted training after the symptoms disappeared.

Every year, all participants answered the medical questionnaire about the competition history, practice frequency, and presence of LBP each year, and underwent MRI. Each participant was fixed in the supine position with the knee joint in mild flexion during the MRI. MRI of the lumbar vertebrae was performed with a flexible quadrature detection body coil on a 0.4 T unit (APERTO, Hitachi Medical, Tokyo, Japan). T2-weighted images in the sagittal and coronal planes were used to assess the characteristic MRI findings. The section thicknesses of the coronal and sagittal views were $3.5 \mathrm{~mm}$, and the interval gaps for both views were $1.0 \mathrm{~mm}$. We checked for lumbar disc degeneration, disc herniation, and spondylolysis at all lumbar vertebral levels (L1-S1) on MRI scans in the sagittal and coronal planes. Lumbar disc degeneration was assessed using the Pfirrmann classification, which proved adequate agreement among 
different observers and by the same observer on separate occasions [9, 10]. MRI findings were interpreted independently by two orthopedic surgeons; one was a specialist in spine surgery (reader 1), and the other was an experienced orthopedic surgeon (reader 2). Each surgeon interpreted the MRI findings twice, and the points of each interpretation were separated by a 2-week period. When the judgments of the two surgeons differed, the two readers consulted and adopted the judgment of the spine surgeon. Inter-reader and intra-reader agreements were assessed using $\mathrm{k}$ values [11]. We defined values $\leq 0$ as indicating no agreement, $0.01-0.20$ as none to slight, $0.21-0.40$ as fair, $0.41-0.60$ as moderate, $0.61-0.80$ as substantial, and $0.81-1.00$ as almost perfect agreement.

\section{Results}

The participants practiced approximately 2 hours per day for around 5 days per week under the guidance of a team coach. Prior to starting this study, 11 of the 12 participants participated in non-weightlifting sports, and two participants participated in a new sport in a 5-year follow-up. There were no positive findings of lumbar disc herniation and spondylolysis on MRI; LBP was also not observed, but grade II disc degeneration changes were detected in two participants at the start of this study.

During this 5-year cohort study, eight participants (67\%) had lumbar disc degeneration in the second year, nine $(75 \%)$ in the third year, and $12(100 \%)$ after the fourth year (Table 2). Lumbar disc degeneration was almost irreversible, and the worst grade was as follows: five of the 12 cases were grade II, two cases were grade III, and five cases were grade IV in the final year. Grade III or IV was the most common at L4/5 (33\%), followed by L5/S (25\%), and grade III or IV was detected in two participants at two-disc levels (Table 3). The $\mathrm{k}$ value of inter-reader agreement was 0.80 (substantial), and intra-reader agreement was 0.70 (substantial, mean of the readers). Lumbar disc herniation findings were detected in four participants (33\%) over 5 years, and one of them underwent herniotomy in 2017.

Lumbar spondylolysis was detected in seven participants (58\%) over 5 years, and two of them were found at the same level for 2 consecutive years. It was most common in L5 (33\%), followed by L3 (17\%). In the final year, abnormal MRI findings were detected in all participants, but only three participants had LBP (Table 3). We present two representative cases of lumbar disc degeneration and spondylolysis, and progressive disc degeneration and disc herniation (Figs. 1 and 2).

\section{Discussion}

No prospective studies have focused on lumbar degeneration in child and adolescent weightlifters before epiphyseal closure, other than our 3-year cohort study [8]. The present 5-year cohort study revealed that long-term continuation of weightlifting training in children and adolescents is associated with the development of lumbar degeneration, especially disc degeneration. This cohort study will help predict future lumbar degeneration in children and adolescent weightlifters and develop safe training strategies. As mentioned earlier, there are negative opinions about training because weightlifting training in children before epiphyseal closure can lead to growth plate disorders; however, recent studies have shown that 
proper weight training under the supervision of a qualified adult is effective in improving performance and preventing injuries [1-3]. Participants in this study were competition-level weightlifters, so supervisors were more likely to have provided weightlifters with safe and appropriate training guidance. However, lumbar disc degeneration was detected in all participants after the fourth year, and disc herniation findings were detected in $33 \%$ of participants in the final years; one of the participants underwent herniotomy. Furthermore, lumbar spondylolysis was detected in $58 \%$ of the patients, but no participants had chronic LBP and nonunion. This result may indicate that lumbar degeneration occurs frequently with long-term weightlifting training in child and adolescent weightlifters, even under the guidance of supervisors.

Lumbar disc degeneration is characterized by the loss of disc hydration, disc space narrowing, and annular tears [4]. Disc degeneration is considered almost irreversible because the lumbar disc has limited regenerative capabilities due to poor vascularity [12], which can lead to other problems, including disc herniation or spondylolysis [13]. Tertti et al. reported that the prevalence of disc degeneration in asymptomatic and symptomatic subjects in a 15 -year-old adolescent was $26 \%$ and $38 \%$, respectively [14]. In addition, Salo et al. reported that the prevalence of disc degeneration in children under the age of 15 years was $22 \%$, and all occurred in children over the age of 10 years [15]. In this study, disc degeneration was detected in $\geq 90 \%$ of patients in the third year, when the average age of the participants was 15 years. Furthermore, the two youngest 8-year-old children at the beginning of this study had disc degeneration from the age of 9 years.

Lumbar disc herniation is a rare disease in children, and the cumulative incidence of disc herniation below 17 years is four in 10,000 [16]. Disc herniation findings were detected in four participants (33\%) over 5 years, with a significantly higher incidence. The development of disc degeneration or disc herniation in children is attributed to competition-level sports participation and lifestyle factors [16], and this might be the result of continued stress on disc degeneration. The most important finding of this study was that disc degeneration progressed from a young age in participants without LBP. Disc degeneration is almost irreversible, and participants continue to be at risk of developing LBP in the future.

Lumbar spondylolysis is considered a stress fracture due to repetitive hyperextension and axial loading of the spine. It occurs more often in young athletes than in adults, particularly in baseball, gymnastics, football, tennis, and weightlifting $[4,17,18]$. In acute spondylolysis, the cure rate is $\geq 90 \%$ with appropriate conservative treatment. However, nonunion of the fractured part due to delayed diagnosis or treatment causes chronic LBP, spondylolisthesis, and sciatica [17-19]. In other words, it is important to detect spondylolysis at an early stage of onset and to provide appropriate conservative treatment. T2weighted MRI is excellent for early indications of spondylolysis, and CT is useful in assessing longitudinal changes in bony union of acute pars defects diagnosed by MRI. Considering these and radiation exposure, MRI is the modality of choice for diagnosing spondylolysis in children, and we defined pedicle signal changes as spondylolysis using MRI in this study $[4,18]$. In this study, seven cases (58\%) of spondylolysis were found in 5 years, but all were in the early stage. Two of these cases showed early stage spondylolysis similar to levels in the following year, but none of them developed chronic LBP or 
nonunion, and no spondylolysis was detected the following year. One case of early stage spondylolysis was found in the final year, and she returned to practice after conservative treatment. It is a well-known fact that spondylolysis is more likely to occur during the growth period $[4,16]$, which is detected in more than half of the participants in the study. A review of child athletes with LBP reported that exercising 5 days or more than 20 hours a week increased the risk of developing spondylolysis [16], and participants in this study corresponded to this. Most importantly, long-term hard weightlifting training during growth is a risk factor for spondylolysis, but irreversible disability can be prevented and return to competition with proper conservative treatment.

This is the first study to prospectively investigate lumbar degeneration in children and adolescent weightlifters. Weightlifting training in children and adolescents requires more careful supervision and long-term follow-up because it frequently causes lumbar degeneration, potentially increasing the risk of developing LBP.

This cohort study had some limitations. First, because few athletes start weightlifting at the competition level from childhood or adolescence, the sample size was small. We plan to continue annual medical examinations, and there is room to evaluate more participants. Second, most participants participated in sports other than weightlifting. Furthermore, two participants started a new sport during the 5-year followup period. Therefore, the results of this study may be attributed to the influence of sports other than weightlifting. Previous studies have reported that various sports, including weightlifting, contribute to lumbar degeneration $[4,18]$, but participants spent most of their time training in weightlifting. Third, this study did not compare participants with controls of similar age groups. Although we do not have unique knowledge of lumbar degeneration in children, the incidence of lumbar degeneration was clearly higher in the participants of this study, based on previous studies [14-16]. Finally, the association between lumbar degeneration detected in participants and the development of LBP in the future remains unclear, and a longer follow-up of participants is required.

In the future, it will be necessary to investigate the relationship between lumbar degeneration and LBP over a longer period of time with a larger sample size. We would like to emphasize that the findings of this study may help to prevent irreversible injuries in children and adolescent athletes undergoing weightlifting training.

\section{Conclusion}

A 5-year cohort study of 12 children and adolescent weightlifters detected lumbar degeneration in all participants. The details of lumbar degeneration were as follows: all (100\%) of the 12 participants had disc degeneration, four (33\%) participants had disc herniation in the 5-year follow-up, and spondylolysis was detected in seven (58\%) participants by the time of the final year. Long-term hard weightlifting training during the growth period may increase the risk of developing LBP in the future as well as in the present. 


\section{Declarations}

Acknowledgements

This study would not have been possible without participants' cooperation.

\section{Author Contributions}

K.K., J.N., K.S., K.A., K.T., and M.K. provided support for the examination. K.Y. scored the MRI images. All authors read and approved the final manuscript.

\section{Competing Interests}

The authors declare that they have no competing interests.

\section{Data Availability}

A part of data generated or analyzed during this study are included in this published article [and its supplementary information files]. The complete datasets used and/or analyzed during the current study are available from the corresponding author on reasonable request.

\section{Ethics Declarations}

The study design was approved by the ethics committee of our institute (approval \#1399). The purpose and potential risks of this study were explained to the subjects, and written informed consent was obtained from all participants.

\section{Consent}

The research participation agreement also included an item of public consent.

\section{References}

1. Faigenbaum, A. D., Lloyd, R. S., MacDonald, J., Myer, G. D. Citius, Altius, Fortius: beneficial effects of resistance training for young athletes: Narrative review. Br J Sports Med. Jan;50(1):3-7, 2016.

2. Fares, M. Y. et al. Low Back Pain Among Weightlifting Adolescents and Young Adults. Jul 11;12(7): e9127, 2020.

3. Milone, M. T., Bernstein, J., Freedman, K. B., Tjoumakaris, F. There is no need to avoid resistance training (weightlifting) until physeal closure. Phys Sports med. Nov;41(4):101-5, 2013.

4. Hsu, W. K., Jenkins, T. J. Management of Lumbar Conditions in the Elite Athlete. J Am Acad Orthop Surg. Jul;25(7):489-498, 2017.

5. van den Heuvel, M. M. et al. The Prevalence of Abnormalities in the Pediatric Spine on MRI: A Systematic Review and Meta-Analysis. Spine (Phila Pa 1976). Sep 15;45(18): E1185-E1196, 2020. 
6. Aggrawal D., Kaur R., Kumar S., Mathur D. N. A study of changes in the spine in weight lifters and other athletes. Br J Sports Med. Jun;13(2):58-61, 1979.

7. Baranto A., Hellström M., Cederlund C. G., Nyman R., Swärd L. Back pain and MRI changes in the thoraco-lumbar spine of top athletes in four different sports: a 15-year follow-up study. Knee Surg Sports Traumatol Arthrosc. Sep;17(9):1125-34, 2009.

8. Shimozaki K. et al. Incidence rates and characteristics of abnormal lumbar findings and low back pain in child and adolescent weightlifter: A prospective three-year cohort study. PLoS One. Oct 29;13(10): e0206125, 2018.

9. Pfirrmann C. W., Metzdorf A., Zanetti M., Hodler J., Boos N. Magnetic resonance classification of lumbar intervertebral disc degeneration. Spine (Phila Pa 1976). Sep 1;26(17):1873-8,

10. Urrutia J. et al. The Pfirrmann classification of lumbar intervertebral disc degeneration: an independent inter- and intra-observer agreement assessment. Eur Spine J. Sep;25(9):2728-33, 2016.

11. Kundel H. L., Polansky M. Measurement of observer agreement. Radiology. Aug;228(2):303-8, 2003.

12. Nishida K. et al. Modulation of the biologic activity of the rabbit intervertebral disc by gene therapy: an in vivo study of adenovirus-mediated transfer of the human transforming growth factor beta 1 encoding gene. Spine (Phila Pa 1976). Dec 1;24(23):2419-25, 1999.

13. Chan S. C., Ferguson S. J., Wuertz K., Gantenbein-Ritter B. Biological response of the intervertebral disc to repetitive short-term cyclic torsion. Spine (Phila Pa 1976). Nov 15;36(24):2021-30, 2011.

14. Tertti M. O., Salminen J. J., Paajanen H. E., Terho P. H., Kormano M. J. Low-back pain and disk degeneration in children: a case-control MR imaging study. Aug;180(2):503-7, 1991.

15. Salo S., Paajanen H., Alanen A. Disc degeneration of pediatric patients in lumbar MRI. Pediatr Radiol. 25(3):186-9, 1995.

16. Raghu A. L. B., Wiggins A., Kandasamy J. Surgical management of lumbar disc herniation in children and adolescents. Clin Neurol Neurosurg. Oct;185:105486, 2019.

17. Campbell R. S., Grainger A. J., Hide I. G., Papastefanou S., Greenough C. G. Juvenile spondylolysis: a comparative analysis of CT, SPECT and MRI. Skeletal Radiol. Feb;34(2):63-73, 2005.

18. Goetzinger S et al. Spondylolysis in Young Athletes: An Overview Emphasizing Nonoperative Management. J Sports Med (Hindawi Publ Corp). Jan 21;2020:9235958, 2020.

19. Klein G., Mehlman C. T, McCarty M. Nonoperative treatment of spondylolysis and grade I spondylolisthesis in children and young adults: A meta-analysis of observational studies. J Pediatr Orthop. Mar;29(2):146-56, 2009.

\section{Tables}

Due to technical limitations, table 1, 2 and 3 is only available as a download in the Supplemental Files section.

\section{Figures}




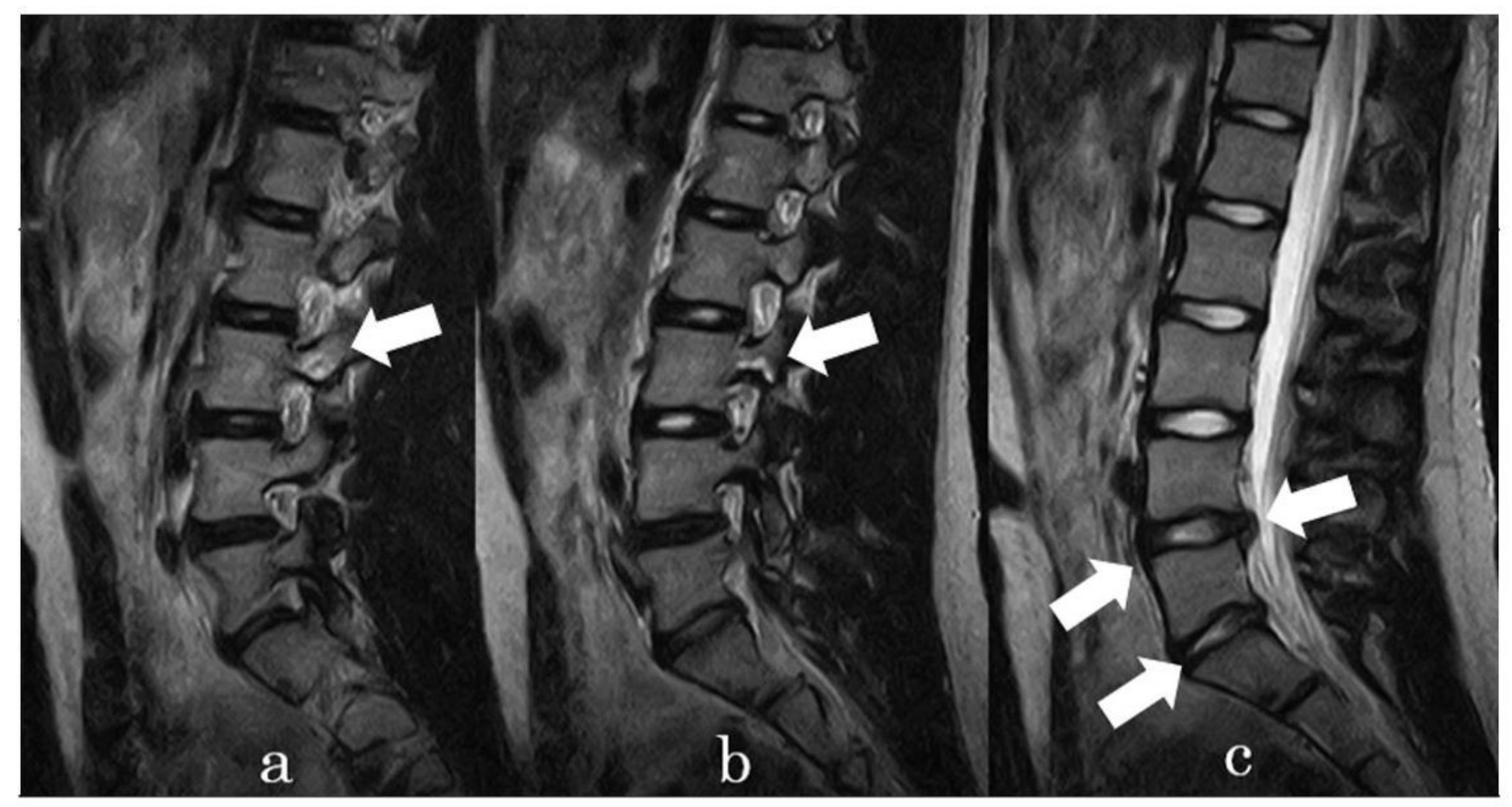

Figure 1

Representative case (patient 7) with bilateral lumbar spondylolysis at L3. (a, b) Disc degeneration at L4/5. L5/S1. (c) Disc herniation at L4/5.

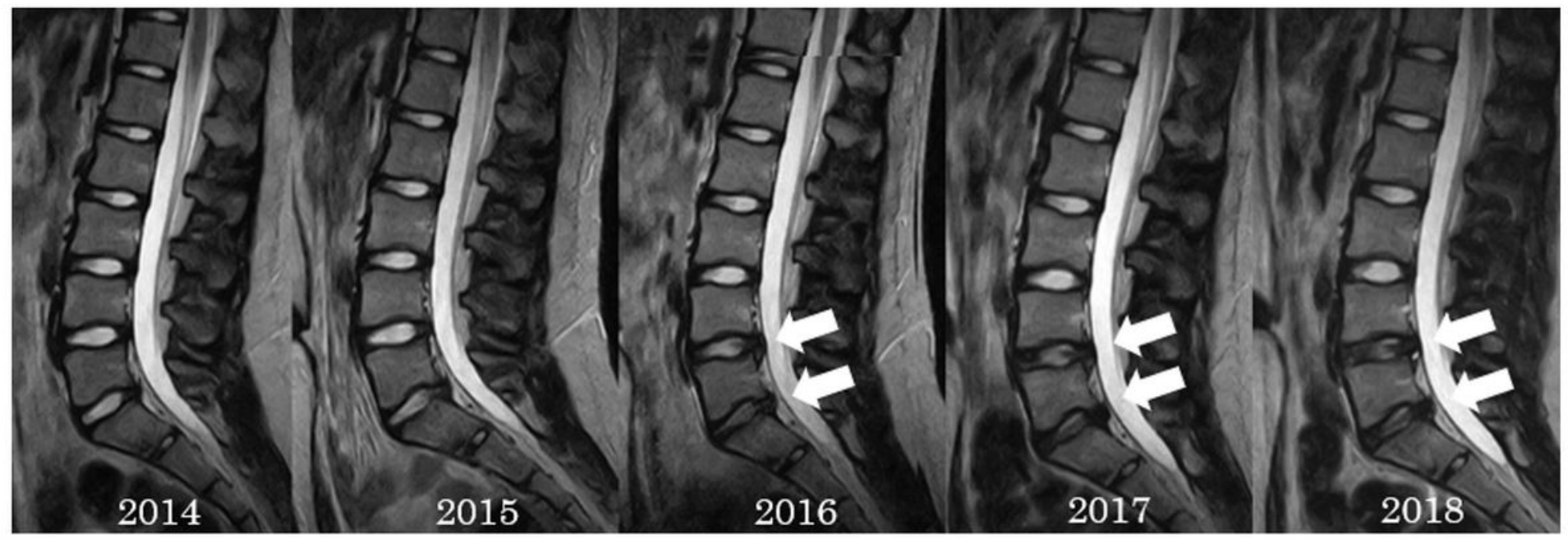

Figure 2

Representative case (patient 8). There were no findings in 2014. From 2016, there was progressive lumbar disc degeneration and disc herniation at L4/5 and L5/S1.

\section{Supplementary Files}


This is a list of supplementary files associated with this preprint. Click to download.

- Table1.xlsx

- Table2.xlsx

- Table3.xlsx 